Ciência Florestal, Santa Maria, v. 21, n. 2, p. 315-322, abr.-jun., 2011

\title{
ESTUDO DA MASSA ESPECÍFICA BÁSICA E DA VARIAÇ̃̃O DIMENSIONAL DA MADEIRA DE TRÊS ESPÉCIES DE EUCALIPTO PARA A INDÚSTRIA MOVELEIRA
}

\section{SPECIFIC GRAVITY AND SHRINKAGE OF WOOD OF THREE SPECIES OF EUCALYPTS FOR FURNITURE PRODUCTION}

\author{
Camila Santos Doubek Lopes ${ }^{1}$ Adriana Maria Nolasco ${ }^{2}$ Mário Tomazello Filho ${ }^{3}$ \\ Carlos Tadeu dos Santos Dias ${ }^{4}$ Alexandre Pansini ${ }^{5}$
}

\begin{abstract}
RESUMO
A utilização da madeira de árvores de espécies de reflorestamento pela indústria moveleira tem apresentado tendência de crescimento nos últimos anos. Nesse sentido, no presente trabalho foram determinadas a massa específica básica e variação dimensional da madeira de árvores de Eucalyptus grandis, Eucalyptus dunnii e Eucalyptus urophylla, de 18 anos, para a aplicação como componentes de madeira maciça em movelaria. Os ensaios laboratoriais para a avaliação da massa específica básica e da variação dimensional da madeira nos sentidos radial e longitudinal do tronco das árvores de eucaliptos foram realizados de acordo com a NBR 7190/96. Os resultados da análise da madeira das espécies de eucalipto foram submetidos ao Teste de Homogeneidade, Análise de Variância, Teste de Tukey e Correlação de Pearson e comparados com o desempenho da madeira de sucupira (Bowdichia nitida) e de cumaru (Dipteryx odorata) utilizadas na indústria moveleira. Verificou-se para a madeira de Eucalyptus grandis menor valor de variação dimensional, sendo a mais indicada para componentes de móveis que exigem elevada estabilidade dimensional, além de peças de maior superfície. A madeira dessa espécie apresentou índice de variação dimensional compatível com o de espécies nativas utilizadas na indústria moveleira. Verificou-se, ainda, variação radial das propriedades da madeira, com alta correlação entre a massa específica básica e a variação dimensional. No sentido longitudinal, a base do tronco das árvores de eucalipto mostrou-se com a região de madeira de maior estabilidade dimensional.
\end{abstract}

Palavras-chave: eucalipto; massa específica básica; retratibilidade; movelaria.

\begin{abstract}
The utilization of wood from reforested species by the furniture industry is a recent trend. Thus, the present study determined the specific gravity and shrinkage of wood of 18-year-old Eucalyptus grandis, Eucalyptus dunnii and Eucalyptus urophylla, for use as components in solid wood furniture making. The tests to evaluate the specific gravity and shrinkage of wood in the radial and axial variation of the eucalyptus trees were performed according to NBR 7190/96. The results of the analysis of wood from eucalypt species were subjected to the Homogeneity Test, ANOVA, Tukey and Pearson correlation and compared to the performance of sucupira wood (Bowdichia nitida) and cumaru wood (Dipteryx odorata), often used in the
\end{abstract}

1. Desenhista Industrial, Mestre, SENAC/SP, Rua Mapuá, 110, CEP 04647-030, São Paulo (SP). camila.sdlopes@sp.senac.br

2. Economista Doméstica, Dra ${ }^{\mathrm{a}}$, Professora do Departamento de Ciência Florestais, Escola Superior de Agricultura Luiz de Queiróz, Universidade de São Paulo, Av. Pádua Dias, 11, Caixa Postal 9, CEP 13418-900, Piracicaba (SP). amnolasc@esalq.usp.br

3. Engenheiro Florestal, Professor Titular do Departamento de Ciências Florestais, Escola Superior de Agricultura Luiz de Queiróz, Universidade de São Paulo, Av. Pádua Dias, 11, Caixa Postal 9, CEP 13418-900, Piracicaba (SP). mtomazel@esalq.usp.br

4. Engenheiro Agrônomo, Professor Titular do Departamento de Ciências Exatas, Escola Superior de Agricultura Luiz de Queiróz, Universidade de São Paulo, Av. Pádua Dias, 11, Caixa Postal 9, CEP 13418-900, Piracicaba (SP). ctsdias@esalq.usp.br

5. Acadêmico do Curso de Engenharia Florestal, Escola Superior de Agricultura Luiz de Queiróz, Universidade de São Paulo, Av. Pádua Dias, 11, Caixa Postal 9, CEP 13418-900, Piracicaba (SP). apansini@esalq.usp.br

Recebido para publicação em 19/05/2008 e aceito em 24/09/2010

Ci. Fl., v. 21, n. 2, abr.-jun., 2011 
furniture industry. The following results were found: Eucalyptus grandis had a lower value of shrinkage, being more suitable for furniture components that require high dimensional stability, as well as parts of larger surface. The wood of this species showed a rate of dimensional variation compatible with the native species used in the furniture industry. The radial variation of the wood was also verified, and a high correlation between specific gravity and shrinkage was found. Longitudinally, the base of the trunk of the eucalyptus trees was shown to be the region of greatest dimensional stability.

Keywords: eucalypts; specific gravity; shrinkage; furniture.

\section{INTRODUÇ̃̃O}

Nos últimos anos, verifica-se um gradativo aumento da utilização da madeira de árvores de plantações florestais de ciclo curto e de alta produtividade, especialmente as dos gêneros Eucalyptus e Pinus, pela indústria moveleira no País. Por outro lado, ocorre uma redução na exploração das árvores das espécies nativas da floresta tropical Amazônica, caracterizadas pela reduzida taxa de crescimento anual e baixa frequência, pela efetividade da aplicação de medidas de fiscalização e controle do desmatamento (OLIVEIRA, 1997).

A literatura reporta algumas características das árvores de eucalipto, como tronco retilíneo, desrama natural e formação de madeira com variações de densidade, coloração, textura e desenho, importantes para a aplicação em movelaria (CALORI e KIKUTI, 1997). No entanto, a madeira de eucalipto utilizada na fabricação de móveis apresenta, de modo geral, características tecnológicas consideradas inapropriadas considerando que provêm de plantações florestais destinadas às indústrias de celulose, chapas, carvão, etc. (LIMA, 2005).

Apesar das vantagens da utilização da madeira de eucalipto, em 2006, somente $3,2 \%$ da produção total foi destinada a serraria (ABRAF, 2007). Nesse aspecto, são necessárias pesquisas para a seleção de espécies potenciais de eucalipto com características silviculturais e tecnológicas da madeira direcionadas para a aplicação em movelaria (PONCE, 1995).

Considera-se que, dos parâmetros físicos de qualidade da madeira, a massa específica básica é um dos mais importantes e relaciona-se à estrutura anatômica e composição química (HILLIS e BROWN, 1984; SCANAVACA e GARCIA, 2004; TRUGILHO et al., 2005), incluindo a resistência mecânica, estabilidade dimensional e qualidade da superfície usinada (LOPES, 2007). A variação dimensional da madeira, da mesma forma, constitui- se em importante parâmetro da madeira para aplicação em movelaria, relacionada à disposição dos raios na seção longitudinal radial e pelo ângulo das microfibrilas na parede das fibras (SILVA e OLIVEIRA, 2003). De acordo com Trugilho et al. (2002) a massa específica básica e a variação dimensional da madeira das espécies florestais apresentam correlação positiva, sendo que as de maior massa de madeira/volume apresentam maior variação dimensional.

Pelo exposto, o presente trabalho teve como objetivos a caracterização e a correlação da massa específica básica e variação dimensional da madeira de árvores de Eucalyptus grandis, Eucalyptus urophylla e Eucalyptus dunnii, aos 18 anos, visando à sua utilização pela indústria de móveis.

\section{MATERIAL E MÉTODOS}

\section{Espécies de eucalipto e caracterização do local}

Foram selecionadas e cortadas três árvores/ espécie de Eucalyptus grandis, Eucalyptus dunnii e de Eucalyptus urophylla de plantações florestais, com 18 anos, da Estação Experimental de Anhembi, SP, do Departamento de Ciências Florestais da ESALQ/USP. As coordenadas geográficas do local são latitude de $22^{\circ} 47^{\prime} \mathrm{S}$, longitude de $48^{\circ} 09^{\prime}$ $\mathrm{W}$ e altitude de $500 \mathrm{~m}$, com relevo suavemente ondulado, solo podzólico arenoso e profundo. O clima é caracterizado como CWA, com verão quente e chuvoso e inverno moderadamente seco e frio, temperatura média anual de $21^{\circ} \mathrm{C}$ e a média do mês mais frio e quente de 17,1 e $23,7^{\circ} \mathrm{C}$ respectivamente. A precipitação anual é de $1.350 \mathrm{~mm}$, com déficit hídrico de $20 \mathrm{~mm}$.

\section{Amostragem e preparo dos corpos-de-prova de madeira}

Foram cortados dois discos do lenho na base, 50 e $100 \%$ do tronco das árvores das três espécies de eucalipto, armazenados por 7 meses em condições de laboratório para a secagem ao ar até atingir a umidade de equilíbrio. Na seção transversal 
de um disco de lenho, foram demarcados e cortados corpos de prova (dimensões de $2 \times 2 \times 8 \mathrm{~cm}$ ) a 0,33 , 66 e $100 \%$ do raio, de acordo com as Normas NBR 7190/96, obtendo-se 72 corpos de prova de madeira/ espécie (Figura 1). O segundo disco de lenho foi utilizado na determinação da variação longitudinal da massa específica básica ao longo do tronco das árvores das três espécies de eucalipto.

I= linha de corte da serra

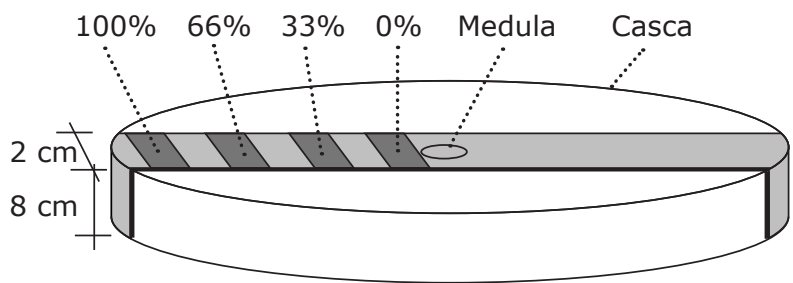

FIGURA 1: Demarcação e dimensões dos corposde-prova de madeira a diferentes porcentagens do raio.

FIGURE 1: Delineation and dimensions of the wood samples in different percentages of the radius.

\section{Determinação da massa específica básica e variação dimensional dos corpos de prova de madeira}

De acordo com a Norma NBR 7190/96, aferindo-se as dimensões dos corpos-de-prova de madeira, pela demarcação de pontos nos planos longitudinal, tangencial, radial para a padronização das mensurações.

\section{Análise estatística dos resultados}

Os resultados da avaliação das dimensões dos corpos de prova de madeira foram analisados (teste de homogeneidade de variância, outliers, escala e tamanho da amostra), com a transformação da retração radial em (log na base 10) e a massa específica básica em $\left(\mathrm{x}^{2}\right)$. Aplicaram-se a análise de variância e o teste de Tukey ajustado para o nível descritivo, significância de 0,05. A interação foi modelada considerando o efeito de espécie (3), posições radial (4), longitudinal (3), em um ensaio fatorial 3:4:3. Posteriormente, aplicaram-se a Correlação de Pearson e as análises no sistema computacional SAS "Statical Analysis System", Versão 9.3.1 (SAS, 2003).

\section{RESULTADOS E DISCUSSÃO}

\section{Massa específica básica da madeira}

Os valores médios de massa específica básica do lenho foram superiores nas árvores de Eucalyptus urophylla, seguindo-se as do Eucalyptus dunnii e Eucalyptus grandis (Tabela 1) sendo concordantes com os apresentados na literatura (OLIVEIRA, 1997; CALORI e KIKUTI, 1997; ROCHA, 2000; GONÇALEZ et al., 2006).

$\mathrm{Na}$ direção radial, verifica-se um gradativo aumento dos valores de massa específica básica dos corpos de prova de madeira das espécies de eucalipto, caracterizando formação da madeira juvenil na região central (próxima a medula) e adulta (próxima à casca). Esse modelo de variação é amplamente retratado por inúmeros autores

TABELA 1: Massa específica básica média da madeira nos sentidos radial e longitudinal do tronco de árvores de Eucalyptus dunnii, Eucalyptus urophylla e Eucalyptus grandis.

TABLE 1: Mean wood basic density in radial and longitudinal direction of the trunk of Eucalyptus dunnii, Eucalyptus urophylla and Eucalyptus grandis trees.

\begin{tabular}{|c|c|c|c|c|c|c|c|c|c|c|}
\hline \multirow{2}{*}{\multicolumn{2}{|c|}{ Tratamento }} & \multicolumn{3}{|c|}{ Eucalyptus dunnii } & \multicolumn{3}{|c|}{ Eucalyptus urophylla } & \multicolumn{3}{|c|}{ Eucalyptus grandis } \\
\hline & & \multirow{2}{*}{$\begin{array}{c}\begin{array}{c}\text { Média } \\
(\mathrm{g} / \mathrm{cm} 3)\end{array} \\
0,53\end{array}$} & \multirow[b]{2}{*}{ b } & \multirow{2}{*}{$\frac{\text { CV (\%) }}{11,79}$} & \multirow{2}{*}{$\begin{array}{c}\begin{array}{c}\text { Média } \\
(\mathrm{g} / \mathrm{cm} 3)\end{array} \\
0,51\end{array}$} & \multirow{2}{*}{\multicolumn{2}{|c|}{\begin{tabular}{|c|} 
CV (\%) \\
3,97
\end{tabular}}} & \multirow{2}{*}{$\begin{array}{c}\begin{array}{c}\text { Média } \\
(\mathrm{g} / \mathrm{cm} 3)\end{array} \\
0,47\end{array}$} & & \multirow{2}{*}{$\frac{\text { CV (\%) }}{9,56}$} \\
\hline \multirow{4}{*}{ 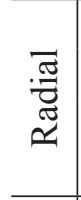 } & $0 \%$ & & & & & & & & c & \\
\hline & $33 \%$ & 0,56 & $\mathrm{~b}$ & 8,59 & 0,71 & $\mathrm{~b}$ & 6,97 & 0,55 & $\mathrm{~b}$ & 11,10 \\
\hline & $66 \%$ & 0,64 & $\mathrm{a}$ & 4,97 & 0,79 & $\mathrm{a}$ & 4,39 & 0,64 & $\mathrm{a}$ & 8,61 \\
\hline & $100 \%$ & 0,69 & $\mathrm{a}$ & 4,67 & 0,75 & $a b$ & 2,56 & 0,67 & $\mathrm{a}$ & 5,74 \\
\hline \multirow{4}{*}{ 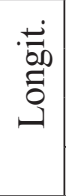 } & Base & 0,59 & $\mathrm{a}$ & 15,47 & 0,71 & $\mathrm{a}$ & 16,91 & 0,59 & $\mathrm{a}$ & 15,01 \\
\hline & $50 \%$ & 0,62 & $\mathrm{a}$ & 10,31 & 0,68 & a & 17,51 & 0,58 & $\mathrm{a}$ & 17,68 \\
\hline & $100 \%$ & 0,61 & $\mathrm{a}$ & 12,82 & 0,69 & $\mathrm{a}$ & 15,41 & 0,57 & $\mathrm{a}$ & 16,49 \\
\hline & Geral & 0,61 & & 12,71 & 0,69 & & 16,27 & 0,58 & & 15,99 \\
\hline
\end{tabular}

Médias seguidas da mesma letra nas colunas não diferem estatisticamente pelo teste de Tukey ajustado para o nível descritivo, com significância de $5 \%(\mathrm{P}<0,05)$; $\mathrm{CV}=$ coeficiente de variação. 
para a madeira de diferentes espécies de eucalipto (PANSHIN e DE ZEEUW, 1980; HILLIS e BROWN, 1984; TOMAZELLO FILHO, 1985; SHIMOYAMA e BARRICHELLO, 1991; LIMA, 1996; OLIVEIRA, 1997; TRUGILHO et al., 2002; SILVA et al., 2005; GONÇALES, et al 2006). Constatou-se uma redução da massa específica básica do lenho localizado na região do alburno das árvores de Eucalyptus urophylla, pelo menor teor de extrativos, corroborando os resultados obtidos por outros autores (LOPES e TOMAZELLO FILHO, 2006).

A massa específica básica da madeira não mostrou variação significativa ao longo da altura do tronco das árvores das três espécies de eucalipto bem como para as médias dentro das espécies (Tabela 1), conforme indicado por alguns autores na literatura especializada (CALORI e KIKUTI, 1997; LIMA, 2005). No entanto, outras tendências são mencionadas, da mesma forma, para outras espécies de eucalipto (OLIVEIRA, 1997; TRUGILHO et al., 2002; SILVA, 2002; SANTOS et al., 2004; GONÇALES, et al 2006).

A massa específica básica da madeira constitui-se em parâmetro tecnológico relacionado com a variação dimensional, resistência mecânica e qualidade da superfície usinada, sendo importante na utilização da madeira para a confecção de móveis de qualidade. Das três espécies, a madeira de Eucalyptus urophylla apresentou maior massa específica básica indicando que, em termos gerais, as características de maior variação dimensional, maior resistência mecânica e melhor acabamento superficial.

\section{Variação dimensional da madeira}

\section{Retração tangencial da madeira}

Não foram verificadas diferenças significativas entre as médias dos valores de retração tangencial da madeira das três espécies de eucalipto, no sentido radial (Tabela 2), sendo concordantes com os apresentados na literatura (OLIVEIRA, 1997; SILVA, 2002; SCANAVACA e GARCIA, 2004; GONÇALES, et al 2006). Observa-se, no entanto, a tendência de aumento do valor da retração tangencial no lenho de Eucalyptus grandis, embora com redução na região da casca para o Eucalyptus dunnii e Eucalyptus urophylla, relatado na bibliografia (TOMAZELLO FILHO, 1985; SHIMOYAMA e BARRICHELLO, 1991; OLIVEIRA, 1997; SILVA, 2002; SILVA e OLIVEIRA, 2003; REZENDE e CRUZ et al., 2003 apud LIMA, 2005; SILVA et al., 2005; LOPES e TOMAZELLO FILHO, 2006). Essa tendência, segundo Silva e Oliveira (2003) deve-se à redução do ângulo microfibrilar, aumento do teor de celulose na parede celular e aumento do comprimento das fibras.

$\mathrm{O}$ valor da retratibilidade tangencial das amostras de madeira ao longo do tronco das árvores das três espécies de eucalipto não mostrou diferença significativa ao nível de significância de 0,05 pelo

TABELA 2: Retração tangencial média da madeira nos sentidos radial e longitudinal do tronco de árvores de Eucalyptus dunnii, Eucalyptus urophylla e Eucalyptus grandis.

TABLE 2: Mean wood tangential shrinkage in radial and longitudinal direction of the trunk of Eucalyptus dunnii, Eucalyptus urophylla and Eucalyptus grandis trees.

\begin{tabular}{|c|c|c|c|c|c|c|c|c|c|c|}
\hline \multirow{2}{*}{\multicolumn{2}{|c|}{ Tratamento }} & \multicolumn{3}{|c|}{ Eucalyptus dunnii } & \multicolumn{3}{|c|}{ Eucalyptus urophylla } & \multicolumn{3}{|c|}{ Eucalyptus grandis } \\
\hline & & Média (\%) & & CV (\%) & Média (\%) & & CV (\%) & Média (\%) & & CV (\%) \\
\hline \multirow{4}{*}{ 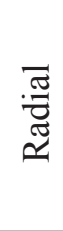 } & 0 & 13,76 & $\mathrm{a}$ & 13,14 & 11,94 & $\mathrm{a}$ & 12,61 & 7,65 & $\mathrm{a}$ & 15,81 \\
\hline & $33 \%$ & 14,76 & $\mathrm{a}$ & 12,50 & 12,03 & $\mathrm{a}$ & 9,55 & 8,16 & $\mathrm{a}$ & 14,12 \\
\hline & $66 \%$ & 14,89 & $\mathrm{a}$ & 15,87 & 12,45 & $\mathrm{a}$ & 6,95 & 8,49 & a & 15,17 \\
\hline & $100 \%$ & 12,92 & $\mathrm{a}$ & 13,43 & 10,87 & $\mathrm{a}$ & 6,95 & 9,22 & $\mathrm{a}$ & 13,13 \\
\hline \multirow{4}{*}{ 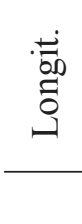 } & Base & 13,88 & $\mathrm{a}$ & 20,35 & 12,16 & $\mathrm{a}$ & 10,31 & 8,02 & $\mathrm{a}$ & 9,83 \\
\hline & $50 \%$ & 14,55 & a & 10,24 & 11,75 & $\mathrm{a}$ & 11,60 & 8,21 & a & 17,53 \\
\hline & $100 \%$ & 13,81 & $\mathrm{a}$ & 11,74 & 11,56 & $\mathrm{a}$ & 8,84 & 8,91 & $\mathrm{a}$ & 16,63 \\
\hline & Geral & 14,08 & & 14,46 & 11,82 & & 10,25 & 8,40 & & 15,51 \\
\hline
\end{tabular}

Médias seguidas da mesma letra nas colunas não diferem estatisticamente pelo teste de Tukey ajustado para o nível descritivo, com significância de 5\% $(\mathrm{P}<0,05)$; CV = coeficiente de variação.

Ci. Fl., v. 21, n. 2, abr.-jun., 2011 
teste de Tukey, corroborando os resultados de Calori e Kikuti (1997).

No entanto, foi detectada diferença estatística entre as médias gerais de retratibilidade tangencial da madeira entre espécies de eucalipto: maior para o Eucalyptus dunnii e menor para o Eucalyptus grandis, indicativa de pior e melhor desempenho respectivamente, considerando a aplicação em movelaria. A retração tangencial da madeira é importante na sua aplicação em movelaria ao indicar a orientação das rachaduras e vãos que podem ocorrer entre as peças de madeira do móvel e que prejudicam os encaixes. Os valores de retratibilidade tangencial de 8,4 e $7,4 \%$ da madeira de cumaru (Dipteryx odorata) e de sucupira (Bowdichia nitida) tradicionalmente empregada em movelaria, são menores aos da madeira de Eucalyptus dunnii e Eucalyptus urophylla, embora similar para o Eucalyptus grandis. A alta retratibilidade da madeira das três espécies de eucalipto é documentada por Oliveira (1997), sendo que o menor e o maior valor de coeficiente de variação para o Eucalyptus urophylla e Eucalyptus grandis, respectivamente, indicam madeira com diferentes níveis de homogeneidade.

\section{Retração radial da madeira}

$\mathrm{O}$ valor de retração radial da madeira de Eucalyptus urophylla foi 28,3 e $56,9 \%$ superior em relação a do Eucalyptus dunnii e Eucalyptus grandis, respectivamente (Tabela 3), estando em conformidade com os apresentados na literatura (CALORI e KIKUTI, 1997; OLIVEIRA, 1997; ROCHA, 2000; SILVA, 2002; SCANAVACA e GARCIA, 2004; GONÇALEZ et al., 2006), a exceção da madeira do Eucalyptus grandis com valor médio inferior.

Das três espécies de eucalipto, somente a madeira do Eucalyptus dunnii apresentou diferença significativa da retração radial a partir de $66 \%$ do raio. Observa-se, no entanto, uma tendência no aumento do valor da retração radial da madeira, retratada na literatura (TOMAZELLO FILHO, 1985; SHIMOYAMA e BARRICHELLO, 1991; OLIVEIRA, 1997; SILVA, 2002; SILVA e OLIVEIRA, 2003; CRUZ et al., 2003 apud LIMA, 2005; SILVA et al., 2005; LOPES e TOMAZELLO FILHO, 2006). No sentido longitudinal verificase a tendência de aumento da retração radial da madeira com os menores valores na região basal do fuste das árvores das árvores de eucalipto (Tabela 3). Para esse parâmetro de qualidade da madeira, as árvores de Eucalyptus urophylla e de Eucalyptus grandis mostraram o pior e o melhor desempenho, visando ao seu uso na forma sólida. A retração radial da madeira do cumaru e de sucupira de 5,4 e 4,5\%, respectivamente (IPT, 2002), é similar a da madeira de Eucalyptus dunnii e de Eucalyptus grandis e abaixo da madeira do Eucalyptus urophylla.

TABELA 3: Retração radial média da madeira nos sentidos radial e longitudinal do tronco de árvores de Eucalyptus dunnii, Eucalyptus urophylla e Eucalyptus grandis.

TABLE 3: Mean wood radial shrinkage in radial and longitudinal direction of the trunk of Eucalyptus dunnii, Eucalyptus urophylla and Eucalyptus grandis trees.

\begin{tabular}{|c|c|c|c|c|c|c|c|c|c|c|}
\hline \multirow{2}{*}{\multicolumn{2}{|c|}{ Tratamento }} & \multicolumn{3}{|c|}{ Eucalyptus dunnii } & \multicolumn{3}{|c|}{ Eucalyptus urophylla } & \multicolumn{3}{|c|}{ Eucalyptus grandis } \\
\hline & & Média (\%) & & $\mathrm{CV}(\%)$ & Média (\%) & & $\mathrm{CV}(\%)$ & Média (\%) & & CV (\%) \\
\hline \multirow{4}{*}{$\begin{array}{l}\widetilde{\widetilde{Z}} \\
\widetilde{\widetilde{Z}} \\
\end{array}$} & 0 & 5,07 & b & 15,50 & 6,98 & a & 14,05 & 4,67 & $\mathrm{a}$ & 20,34 \\
\hline & $33 \%$ & 5,51 & $b$ & 12,29 & 7,76 & $\mathrm{a}$ & 18,30 & 4,31 & a & 17,19 \\
\hline & $66 \%$ & 6,10 & $a b$ & 18,46 & 7,84 & a & 9,54 & 4,80 & $\mathrm{a}$ & 19,78 \\
\hline & $100 \%$ & 6,96 & $\mathrm{a}$ & 20,82 & 7,76 & a & 10,36 & 5,54 & $\mathrm{a}$ & 19,46 \\
\hline \multirow{4}{*}{ 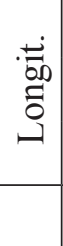 } & Base & 5,63 & $\mathrm{a}$ & 10,80 & 6,80 & $\mathrm{a}$ & 12,05 & 4,49 & $\mathrm{a}$ & 18,87 \\
\hline & $50 \%$ & 6,17 & $\mathrm{a}$ & 26,70 & 7,91 & $\mathrm{~b}$ & 12,35 & 4,61 & $\mathrm{a}$ & 22,69 \\
\hline & $100 \%$ & 5,92 & $\mathrm{a}$ & 21,68 & 8,04 & $\mathrm{~b}$ & 11,11 & 5,39 & $\mathrm{a}$ & 17,42 \\
\hline & Geral & 5,91 & & 21,01 & 7,58 & & 13,72 & 4,83 & & 20,79 \\
\hline
\end{tabular}

Médias seguidas da mesma letra nas colunas não diferem estatisticamente pelo teste de Tukey ajustado para o nível descritivo, com significância de 5\% $(\mathrm{P}<0,05)$; CV $=$ coeficiente de variação. 


\section{Retração volumétrica da madeira}

$\mathrm{O}$ valor médio da retração volumétrica da madeira do Eucalyptus dunnii, Eucalyptus urophylla e Eucalyptus grandis foi de 21,3, 20,7 e $14,1 \%$, respectivamente (Tabela 4). A literatura especializada (OLIVEIRA, 1997; CALORI e KIKUTI, 1997; ROCHA，2000; SILVA，2002; SCANAVACA e GARCIA, 2004; GONÇALEZ et al., 2006) indica valor de retração volumétrica da madeira similar a obtida para o Eucalyptus urophylla, superior a do Eucalyptus dunnii e inferior a relatada para o Eucalyptus grandis.

No sentido radial, observou-se a tendência de aumento do valor da retração volumétrica da madeira, seguido de redução na região do alburno, de 27,7 e 6,6\% em Eucalyptus dunnii e Eucalyptus urophylla (Tabela 4) pela presença de vasos abertos e menor teor de extrativos, corroborando informações de Lopes e Tomazello Filho (2006). $\mathrm{O}$ modelo de variação radial observado para a madeira das 3 espécies de eucalipto é amplamente relatado na bibliografia (TOMAZELLO FILHO, 1985; SHIMOYAMA e BARRICHELLO, 1991; OLIVEIRA, 1997; SILVA, 2002; SILVA e OLIVEIRA, 2003; CRUZ et al., 2003 apud LIMA, 2005; SILVA et al., 2005; LOPES e TOMAZELLO FILHO, 2006), a exceção de alguns autores (ROCHA, 2000) que obtiveram em árvores de Eucalyptus grandis e Eucalyptus dunnii uma redução da retração volumétrica da madeira.

A variação da retração volumétrica da madeira no sentido longitudinal indica que os menores valores estão presentes na base do tronco das árvores das três espécies de eucalipto (Tabela 4), corroborando os resultados das análises realizadas por Trugilho et al. $(2002,2005)$. Comparando-se com a retração volumétrica das madeiras de cumaru e de sucupira, na ordem 13,5 e 12,3\% respectivamente, verificam-se valores superiores para esse parâmetro de qualidade para o Eucalyptus urophylla e Eucalyptus dunnii e inferior para o Eucalyptus grandis, o que o recomenda para a aplicação como peças de mobiliário (IPT, 2002). Segundo Willard (1975) nos móveis de grandes superfícies e de diferentes materiais (ex.: vidro, madeira maciça, laminado, chapas, etc.) avalia-se o desempenho da madeira pelas deformações, com o teor de umidade considerado como importante variável, na qual a contração e o inchamento diferenciado resultam em frestas e trincas superficiais.

Os resultados evidenciam a presença de madeira de menor estabilidade e de maior deformação (contração e inchamento) na região periférica do tronco das árvores das espécies de eucalipto, com as tensões de tração ou de compressão perpendicular às fibras resultando em defeitos como folga, empenamento, trinca de topo, etc. nas peças de madeira dos móveis. Esses defeitos podem romper o filme mecânico, o verniz e, por essa razão, aplicam-se seladoras para o acabamento, evitando a formação de filmes espessos. A redução da instabilidade dimensional da madeira pode ser obtida pela aplicação de ceras, vernizes, lacas e tintas, na umidade de utilização ou de equilíbrio local

TABELA 4: Retração volumétrica média da madeira nos sentidos radial e longitudinal do tronco das árvores de Eucalyptus dunnii, Eucalyptus urophylla e Eucalyptus grandis.

TABLE 4: Mean wood volumetric shrinkage in radial and longitudinal direction of the trunk of Eucalyptus dunnii, Eucalyptus urophylla and Eucalyptus grandis trees.

\begin{tabular}{|c|c|c|c|c|c|c|c|c|c|c|}
\hline \multirow{2}{*}{\multicolumn{2}{|c|}{ Tratamento }} & \multicolumn{3}{|c|}{ Eucalyptus dunnii } & \multicolumn{3}{|c|}{ Eucalyptus urophylla } & \multicolumn{3}{|c|}{ Eucalyptus grandis } \\
\hline & & Média (\%) & & CV (\%) & Média (\%) & & CV (\%) & Média (\%) & & CV (\%) \\
\hline \multirow{4}{*}{ 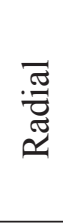 } & 0 & 20,03 & $\mathrm{a}$ & 8,82 & 20,11 & $\mathrm{a}$ & 10,62 & 13,01 & a & 13,67 \\
\hline & $33 \%$ & 21,56 & $\mathrm{a}$ & 10,22 & 20,95 & $\mathrm{a}$ & 7,86 & 13,35 & a & 13,14 \\
\hline & $66 \%$ & 22,20 & $\mathrm{a}$ & 13,95 & 21,52 & $\mathrm{a}$ & 4,58 & 14,18 & $\mathrm{a}$ & 16,20 \\
\hline & $100 \%$ & 21,32 & $\mathrm{a}$ & 14,05 & 20,10 & $\mathrm{a}$ & 9,31 & 15,99 & $\mathrm{a}$ & 14,15 \\
\hline \multirow{4}{*}{ 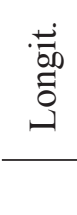 } & Base & 20,67 & $\mathrm{a}$ & 12,87 & 20,17 & $\mathrm{a}$ & 9,61 & 13,44 & $\mathrm{a}$ & 11,86 \\
\hline & $50 \%$ & 22,15 & a & 10,60 & 21,11 & $\mathrm{a}$ & 8,70 & 13,82 & $\mathrm{a}$ & 17,73 \\
\hline & $100 \%$ & 21,02 & $\mathrm{a}$ & 12,97 & 20,73 & a & 7,00 & 15,13 & a & 16,34 \\
\hline & Geral & 21,28 & & 12,17 & 20,67 & & 8,46 & 14,13 & & 16,04 \\
\hline
\end{tabular}

Médias seguidas da mesma letra nas colunas não diferem estatisticamente pelo teste de Tukey ajustado para o nível descritivo, com significância de $5 \%(\mathrm{P}<0,05)$; CV = coeficiente de variação.

Ci. Fl., v. 21, n. 2, abr.-jun., 2011 
dificultando a absorção de umidade (OLIVEIRA, 1997). Considera-se também a aplicação do plano de secagem correto e a seleção de peças de madeira com corte radial são importantes para a obtenção de madeira estável para a fabricação de móveis (WILLARD, 1975).

\section{Correlação de Pearson entre os ensaios}

$\mathrm{O}$ coeficiente de correlação de Pearson avalia o grau e a direção dessa correlação (positiva ou negativa) entre duas variáveis de escala métrica. Dessa forma, a correlação entre a retração tangencial e retração volumétrica da madeira foi a de maior valor (Tabela 5), uma vez que esta é derivada das retrações dos três planos (tangencial, radial e longitudinal). Entre a madeira das três espécies, a de Eucalyptus grandis apresentou resultados significativos para todas as correlações. $\mathrm{O}$ teste mostrou uma baixa correlação significativa (ao nível de 5\%) entre os resultados dos ensaios de retração e de massa específica básica da madeira, significativa somente para a madeira do Eucalyptus grandis. No entanto, o modelo de variação obtido nos ensaios de contração foi coincidente com o da massa específica básica da madeira das espécies de eucalipto e, em concordância com a literatura. A variação dimensional foi de maior magnitude na madeira de maior massa específica básica pela a maior porcentagem de parede celular dos elementos anatômicos e que interagem com a umidade.

TABELA 5: Correlação de Pearson para os valores dos ensaios da madeira de Eucalyptus dunnii, Eucalyptus urophylla e Eucalyptus grandis.

TABLE 5: Pearson correlation for the values of the wood assays for Eucalyptus dunnii, Eucalyptus urophylla and Eucalyptus grandis wood.

\begin{tabular}{c|c|c|c}
\hline Ensaios & $\begin{array}{c}\text { Eucalyptus } \\
\text { dunnii }\end{array}$ & $\begin{array}{c}\text { Eucalyptus } \\
\text { urophylla }\end{array}$ & $\begin{array}{c}\text { Eucalyptus } \\
\text { grandis }\end{array}$ \\
\hline RT . RR & n.s. & n.s. & 0,62 \\
RT . RV & 0,82 & 0,72 & 0,92 \\
RT . MEB & n.s. & n.s. & 0,61 \\
RR . RV & 0,55 & 0,59 & 0,85 \\
RR . MEB & n.s. & n.s. & 0,37 \\
RV . MEB & n.s. & n.s. & 0,61 \\
\hline
\end{tabular}

n.s. - Correlação não significativa ao nível de $5 \%$ de significância. RT - retração tangencial; RR - retração radial; RV - retração volumétrica; MEB - massa específica básica.

\section{CONCLUSÕES}

Os resultados do presente trabalho permitem concluir que, das três espécies de eucalipto, a madeira do Eucalyptus grandis foi a mais estável, com variação dimensional compatível com a da madeira das principais espécies nativas utilizadas pela indústria moveleira. A madeira do Eucalyptus grandis é, dessa forma, indicada para peças que exigem alta estabilidade dimensional e cuja variação de dimensão prejudica o desempenho do móvel. Os índices de variação dimensional e heterogeneidade da madeira das espécies de eucalipto indicam a necessidade de controle nas etapas de desdobro, secagem e acabamento. As características físicas da madeira das espécies de eucalipto variaram significativamente no sentido radial, sendo, dessa forma, importante demarcar a região do lenho com maior massa especifica básica e menor variação dimensional para a produção de peças de mobiliário. No sentido longitudinal, verifica-se, na base do tronco das árvores de eucalipto, a formação de madeira de maior estabilidade dimensional. Ainda, os resultados da análise descritiva indicam que a massa específica básica se constitui em bom indicador da estabilidade dimensional da madeira das três espécies de eucalipto.

\section{REFERÊNCIAS BIBLIOGRÁFICAS}

ABNT - Associação Brasileira de Normas Técnicas. NBR 7190/96: estruturas de madeira. Rio de Janeiro, 1996. 107 p.

ABRAF - Associação Brasileira de Produtores de Florestas Plantadas. Anuário Estatístico da ABRAF 2007 - Disponível em (http://www. abraflor.org.br/estatisticas/anuario-ABRAF-2007. pdf) Acesso em 10 de nov. 2007.

CALORI, J.V.; KIKUTI, P. Propriedades físicas e mecânicas da madeira de Eucalyptus dunnii aos 20 anos de idade. In: IUFRO CONFERENCE ON SILVICULTURA AND IMPROVEMENT ON EUCALYPTS, 1997, Salvador. Proceedings ... Salvador: Embrapa; IPEF; SIF; SEAGRI-DDF, 1997. v. 3, p. 321-326.

GONÇALEZ, J.C. et al. Características tecnológicas da madeira de Eucalyptus grandis W.Hill ex Maiden e Eucalyptus cloeziana F.Muell visando ao seu aproveitamento na indústria moveleira. Ciência Florestal, Santa Maria, v. 16, n. 3, p. 329-341, dez. 2006.

HILLIS, W. E.; BROWN, A. G. Eucalypts for wood 
production. $2^{\text {nd }}$ ed. Melbourne: CSIRO; Academic Press, 1984. 434 p.

IPT - Instituto de Pesquisas Tecnológicas. Madeira para móveis e construção civil. Divisão de produtos florestais. São Paulo, 2002. 1 CD ROM.

LIMA, I. L. Influência do desbaste e da adubação na qualidade da madeira serrada de Eucalyptus grandis Hill ex Maiden. 2005. 161 f. Tese (Doutorado em Tecnologia de Recursos Florestais)Escola Superior de Agricultura "Luiz de Queiroz", Universidade de São Paulo, Piracicaba. 2005.

LOPES, C. S. D. Caracterização da madeira de três espécies de eucalipto para uso em movelaria. 2007. Dissertação (Mestrado em Ciências Florestais)-Escola Superior de Agricultura "Luiz de Queiroz", Universidade de São Paulo, Piracicaba. 2007.

LOPES, C. S. D.; TOMAZELLO FILHO, M. Análise densitométrica da madeira de árvores de Eucalyptus dunnii, de 18 anos de idade. In: SIMPÓSIO DE PÓSGRADUAÇÃO EM CIÊNCIAS FLORESTAIS, 4., 2006, Piracicaba. Anais ... Piracicaba: IPEF, 2006. 1 CD-ROM.

OLIVEIRA, J. T. O. Caracterização da madeira de eucalipto para a construção civil. 1997. Tese (Doutorado em Engenharia) - Escola Politécnica, Universidade de São Paulo, São Paulo, 1997. 2 v.

PANSHIN, A. J.; DE ZEEUW, C. Textbook of wood technology. $4^{\text {th }}$ ed. New York: McGraw Hill, 1980. 722 p.

PONCE, R. H. Madeira serrada de eucalipto: desafios e perspectivas. In: SEMINÁRIO INTERNACIONAL DE UTILIZAÇÃO DE MADEIRA DE EUCALIPTO PARA SERRARIA 1995, São Paulo. Anais... 1995, São Paulo.IPT, IPEF. p.50-58.

ROCHA, M. P. Eucalyptus grandis Hill ex Maiden e Eucalyptus dunnii Maiden como fontes de matéria prima para serraria. 2000. 185 f. Tese (Doutorado em Ciências Florestais) - Universidade Federal do Paraná, Curitiba. 2000.

SANTOS, P. E. T. et al. Posição da tora na árvore e sua relação com a qualidade da madeira serrada de Eucalyptus grandis. Scientia Florestalis, Piracicaba, n. 66, p. 142-151, dez. 2004.

SCANAVACA JUNIOR, L.: GARCIA, J. N. Determinação das propriedades físicas e mecânicas da madeira de Eucalyptus urophylla. Scientia Florestalis, Piracicaba, n. 65, p. 120-129, jun. 2004. SHIMOYAMA, V. R. S.; BARRICHELLO, L. E. G. Influência de características anatômicas e químicas sobre a densidade básica da madeira de Eucalyptus spp. In: CONGRESSO ANUAL DE CELULOSE E PAPEL, 24., 1991, São Paulo. Anais ... São Paulo: ABTCP, 1991. p. 178-183.

SILVA, J. C. Caracterização da madeira de Eucalyptus grandis Hill ex Maiden de diferentes idades, visando sua utilização na indústria moveleira. 2002. 160 f. Tese (Doutorado em Tecnologia e Utilização de Produtos Florestais)Universidade Federal do Paraná, Curitiba, 2002.

SILVA, J. C.; OLIVEIRA, J. T. S. Avaliação das propriedades higroscópicas da madeira de Eucalyptus saligna Sm., em diferentes condições de umidade relativa do ar. Revista Árvore, Viçosa, v. 27, n. 2, p. 233-239, mar./abr. 2003.

SILVA, J. R. M. et al. Influência da morfologia das fibras na usinabilidade da madeira de Eucalyptus grandis Hill ex Maiden. Revista Árvore, Viçosa, v. 29, n. 3, p. 479-487, 2005.

TOMAZELLO FILHO, M. Variação radial da densidade básica e da estrutura anatômica da madeira do Eucalyptus saligna e Eucalyptus grandis. IPEF, Piracicaba, n. 29, p. 37-45, 1985.

TRUGILHO, P. F. et al. Influência da idade nas características do eucalipto. Revista da Madeira, Curitiba, n. 92, p. 82-88, 2005.

TRUGILHO, P. F. et al. Variação dimensional e densidade da madeira em árvore de Eucalyptus. In: ENCONTRO NACIONAL EM MADEIRAS E EM ESTRUTURAS DE MADEIRA, 8., 2002, Uberlândia. Anais... São Carlos: IBRAMEM, 2002.

WILLARD, R. Furniture construction. North Caroline State University, $3^{\text {rd }}$.ed. Raleigh: 1975. $315 \mathrm{p}$. 